\title{
Composição e Diversidade da Fauna de Grilos (Orthoptera: Grylloidea) em um Fragmento de Floresta Pluvial Atlântica do Estado do Rio de Janeiro
}

\author{
Camila Santos Pantoja de Oliveira1, Matheus Prem Mendes², Marcelo Netto Duarte ${ }^{2}$ \& \\ William Costa Rodrigues ${ }^{\circledR} \bowtie$
}

1. Universidade Federal Rural do Rio de Janeiro, Programa de Mestrado em Ciências Veterinárias, e-mail: milapantoja88@yahoo.com.br. 2. Universidade Severino Sombra, e-mail: matheusprem@gmail.com, mnettoduarte@yahoo.com.br. 3. Methodos Consultoria Ambiental Ltda ME e Centro Universitário de Volta Redonda (UniFOA), e-mail: wcrodrigues@ebras.bio.br (Autor para correspondência ${ }^{\bowtie}$ ).

\section{EntomoBrasilis 6 (3): 184-192 (2013)}

Resumo. O objetivo foi analisar a fauna de grilídeos, sua relação com diferentes composições vegetais, o nível de serapilheira eos efeitos da sazonalidade. Foram realizadas quatro coletas, nas quatro estações do ano, entre abril de 2008 e março de 2009. Foram coletados 503 grilídeos com armadilhas de solo, em oito pontos de coleta. Os insetos coletados foram identificados os seguintes gêneros Anurogryllus, Anurogryllus, Argizala, Hygronemobius, Nemobius, Odontogryllus e Pteronemobius. Os grilídeos não possuem afinidade com solos descampados, diminuindo consideravelmente no local mais impactado. O nível de serapilheira não interferiu na abundância e as mudanças sazonais mostraram importância na variação populacional, entretanto, a diversidade e dominância mostraram-se fiéis às alterações ambientais mesmo em menores valores de abundância. Esses resultados mostram que os grilídeos podem ser indicadores ambientais, pois respondem às modificações do meio.

Palavras-chave: Composição Vegetal; Grilos; Insetos; Serapilheira; Sazonalidade.

\section{Composition and Diversity of the Crickets Fauna (Orthoptera: Grylloidea) in a Fragment of Atlantic} Rain Forest in the Rio de Janeiro State

Abstract. This study aimed to analyze the fauna of cricket, its relation with different plant compositions, litter level and seasonality effects. Four collections were performed, in four season of the year, between April 2008 and March 2009. It was collected 503 crickets using soil traps, in eight sampling points. The insects collected were identified as the genera Anurogryllus, Argizala, Hygronemobius, Nemobius, Odontogryllus e Pteronemobius. The crickets have no affinity with uncovered soil, decreasing significantly in more impacted places. The litter level did not affect the abundance and the seasonal changes shown importance in the population variation, however, diversity and dominance showed fidelity to environmental changes even in lower abundance. These results show that crickets may be environmental indicators due to the answers the changes in environment.

Keywords: Cricket; Insects; Litter; Plant Composition; Seasonality.

fragmentação da vegetação provoca mudanças físicas e biológicas nos ambientes naturais como resultado da diminuição de ambientes e do aumento da incidência luminosa (TABANEZ et al. 1997). Os interiores de fragmentos maiores são mais semelhantes entre si quanto à estrutura da vegetação, enquanto as bordas dos fragmentos menores é que se assemelham mais (LiMA-RIBEIRo 2008).

Os insetos são adequados para uso em estudos de avaliação de impacto ambiental e de efeitos de fragmentação florestal, pois, além de ser o grupo de animais mais numeroso do globo terrestre, com elevadas densidades populacionais, apresentam grande diversidade, em termos de espécies e de habitats, e grande variedade de habilidades para dispersão e seleção de hospedeiros e de respostas à qualidade e quantidade de recursos disponíveis, além de sua dinâmica populacional ser altamente influenciada pela heterogeneidade dentro de um mesmo habitat (Thomazin \& Thomazini 2000).

Alguns grupos de insetos, dentre os quais ortopteróides, borboletas e formigas, são especialmente úteis no monitoramento ambiental, principalmente em pequenos fragmentos e áreas com longa história de influência antrópica (Silveira Neto et al. 1995; MAтyot 1998; Freitas et al. 2004). Desta forma, o interesse pelas questões ambientais despertou a necessidade de se ampliar o conhecimento da biodiversidade, bem como, da biologia e da ecologia de cada espécie (LuTINSKI \& GARCIA 2005).
O conhecimento da ortopterofauna da América do Sul está muito aquém do que se tem relatado, especialmente em relação a alguns grupos como, por exemplo, aos Grylloidea e Tettigonioidea. Ainda, da maioria das espécies descritas não se conhecem as necessidades ecológicas, as características comportamentais ou a dinâmica populacional (SAMWAYS \& LOCKWOOD 1998).

Relativamente poucas espécies vivem em regiões temperadas. Ao contrário, nas regiões tropicais são exatamente diversos e estão presentes do nível do solo até o dossel das árvores. Até agora, dados da biologia dos grilos nos seus ambientes naturais são focalizados em poucas espécies que vivem frequentemente na biota não florestal (HubER et al. 1989).

De forma geral, as florestas tropicais são sazonais (MuRPHY \& LUGO 1986) com períodos úmidos de alta produtividade primária e períodos secos menos produtivos (SÁNchez-AzofeIfa et al. 2005; Pezzins et al. 2008). Com isso, WoldA (1978) demonstrou a importância dessas flutuações para a dinâmica das populações e estrutura das comunidades de insetos tropicais. Para SPERBER (1999), os grilos habitantes das florestas tropicais são pouco conhecidos. Estudos taxonômicos, sistemáticos e biológicos são escassos para a maioria dos grupos neotropicais (Mews 2006).

Os grilos são um componente importante da macrofauna de serapilheira florestal (GREENBERG \& FORREST 2003), apresentando predominância de espécies ápteras ou micrópteras (SPERBER et al. 
2003).

Com relação às mudanças nas condições ambientais, os grilos têm limites de tolerância restritos (McCluney \& Date 2008), de tal forma que ambientes em condições menos propícias prejudicam a sobrevivência de muitas espécies (SzINwELSKI 2009). Dessa forma, os mecanismos importantes para a manutenção dos insetos e os efeitos destes no restante da comunidade são aspectos fundamentais a serem considerados em estudos de ecologia (BASSET et al. 2003)

O objetivo desse trabalho foi caracterizar e comparar a composição e a diversidade da fauna de grilos de serrapilheira em diferentes habitats de um fragmento de Floresta Pluvial Atlântica no Estado do Rio de Janeiro.

\section{MATERIAL E MÉTODOS}

O local de estudo foi um fragmento de Mata Atlântica, com área de aproximadamente 19 ha, localizado no Instituto Zoobotânico de Morro Azul (IZMA), município de Engenheiro Paulo de Frontin, RJ, geograficamente situado na lat. $22^{\circ} 29^{\prime} 39.70$ "S e long. $43^{\circ} 34^{\prime} 04.46^{\prime \prime} \mathrm{O}$, com altitude variando entre 671 a $825 \mathrm{~m}$. Neste local existe uma trilha ecológica denominada Trilha dos Quatis (2200 m), onde ao longo desta trilha foram estabelecidos os pontos para a coleta dos insetos (Figura 1).

Foram realizadas quatro coletas abrangendo as quatro períodos distintos ao longo do ano (abril 2008, agosto 2008, outubro 2008 e março 2009). As coletas ocorreram em oito pontos diferentes (I a VIII) com área de $200 \mathrm{~m}^{2}$ cada, ao longo da Trilha dos Quatis. Foram dispostos em seis altitudes distintas, levando em consideração a composição vegetal, o nível de serapilheira (NS) e a luminosidade (Figura 2) (Tabela 1).

Os níveis de serrapilheira forma determinados adotando-se a metodologia do quadrado com 50 × $50 \mathrm{~cm}\left(0,25 \mathrm{~m}^{2}\right)$, seno aplicado três quadrados por ponto de forma aleatória, durante os levantamentos, sendo estimada uma média geral para todos os levantamentos, por ponto de coleta. A medição deu-se utilizando uma régua de alumínio, medindo deste a superfície até o final da serrapilheira, para a medição foi aberta uma pequena trincheira cuidadosamente, para não descaracterizar o local e permitir uma maior precisão. Não foram repetidos pontos de medição ao longo das quatro coletas.

Para a captura dos insetos foram estabelecidos oito pontos com distância entre si irregular, pois a seleção baseou-se nas características da composição vegetal, altitude, cobertura vegetal e nível de serrapilheira (Figura 2). Em cada ponto foram utilizadas seis armadilhas de solo tipo pitfall adaptadas de MAJER (1978) e CASTRo et al. (1989), com altura de $15 \mathrm{~cm}, 12 \mathrm{~cm}$ de diâmetro, com capacidade de $500 \mathrm{~mL}$ de volume (Figura 3), distantes entre si $10 \mathrm{~m}$ em um transecto retangular com $200 \mathrm{~m}^{2}$ (Figura 4). Para fixação foi utilizada uma solução de $150 \mathrm{~mL}$ contendo água (90\%), detergente neutro (10\%) e formol (1\%). Para evitar o transbordamento das armadilhas, devido às precipitações pluviométricas, foi utilizada uma proteção de arame e plástico como cobertura (Figura 5).

Para calcular a área de cobertura do dossel, foram feitas fotografias do dossel, a partir do solo, em cinco pontos aleatórios dentro de áreas dos oito pontos de coleta, utilizando câmera fotográfica Nikon D310o com Lente 18-55 mm, sendo fotografada em cinco pontos aleatórios. O cálculo da área do dossel foi feito através do software Gap Light Analyzer v2.o (FrAzer et al. 1999). Através dos valores da área, obtém a densidade do dossel, para a taxa média de entrada de luz solar por metro quadrado (Tabela 1).

Os insetos coletados foram levados ao Laboratório de Ciências Ambientais da Universidade Severino Sombra, onde foram triados, sendo identificados em nível de família e armazenados em microtubos tipo Eppendorf contendo álcool a $70 \%$. Posteriormente, os indivíduos da família Gryllidae foram determinados em nível de gênero, baseando-se em chaves textuais de Strohecker (1953); Chopard (1938, 1956); Pereira (2012) e chaves pictórias v5.0/5.0 proposta por EADEs et al. (2013).

Os dados foram submetidas à análise estatística, através dos índices de diversidade de Shanon e Dominância e Diversidade de Simpson, além de estimar a Equitatividade J e Hill, para todas as coletas, por ponto e o total por ponto, utilizando o software DivEs - Diversidade de Espécies versão 2.o (RoDRigues 2005).

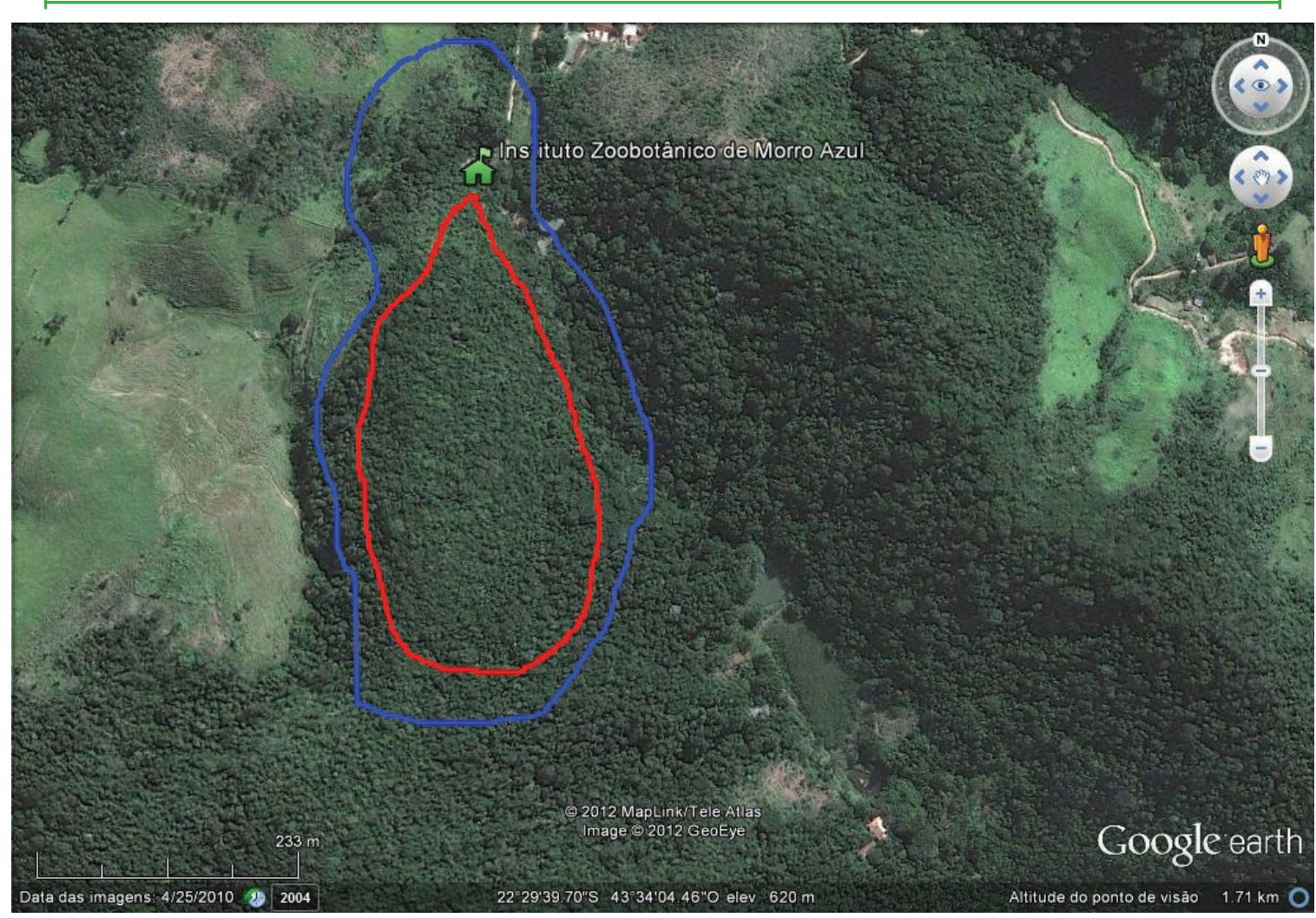

Figura 1. A delimitação em azul indica a área total do IZMA, enquanto a delimitação em vermelho indica a área de estudo onde foram realizadas as coletas (Fonte: Google Earth 2012). 


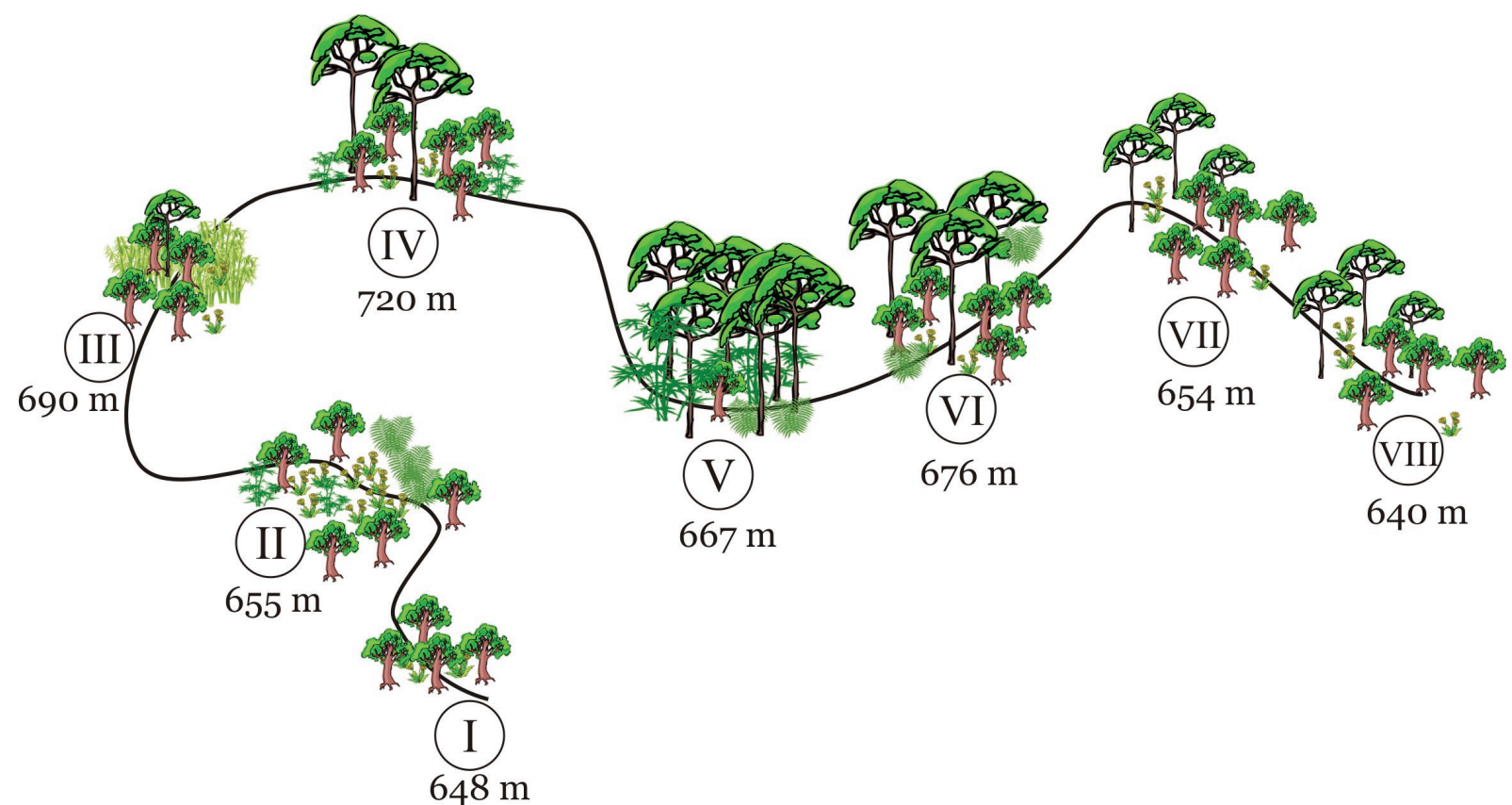

Figura 2. Pontos de coleta e variação da altitude e composição vegetal, em fragmento de floresta pluvial atlântica pertencente ao Instituto Zoobotânico de Morro Azul.

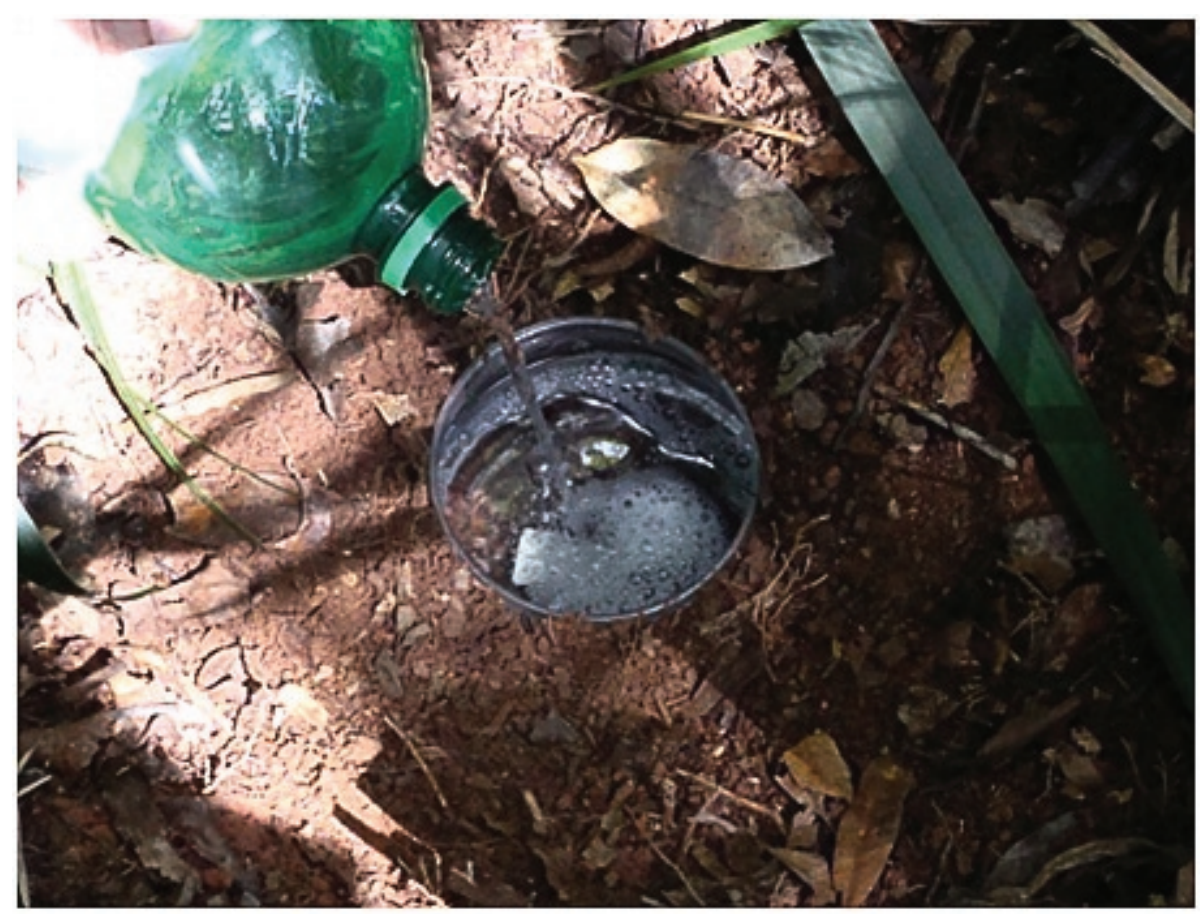

Figura 3: Montagem da armadilha do tipo pitfal, em fragmento de floresta pluvial atlântica pertencente ao Instituto Zoobotânico de Morro Azul.

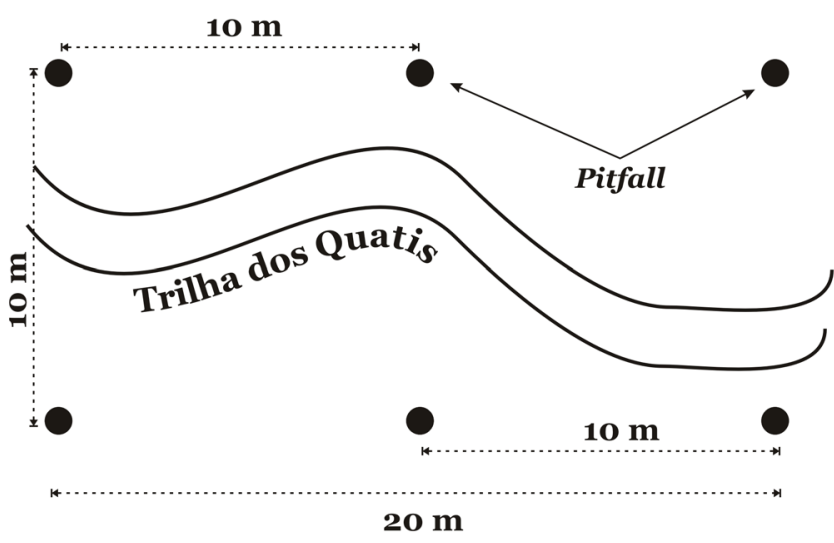

Figura 4. Transecto com a distribuição das armadilhas em cada uma das oito áreas de coleta, com área de $200 \mathrm{~m}^{2}$, em fragmento de floresta pluvial pertencente ao Instituto Zoobotânico de Morro Azul.
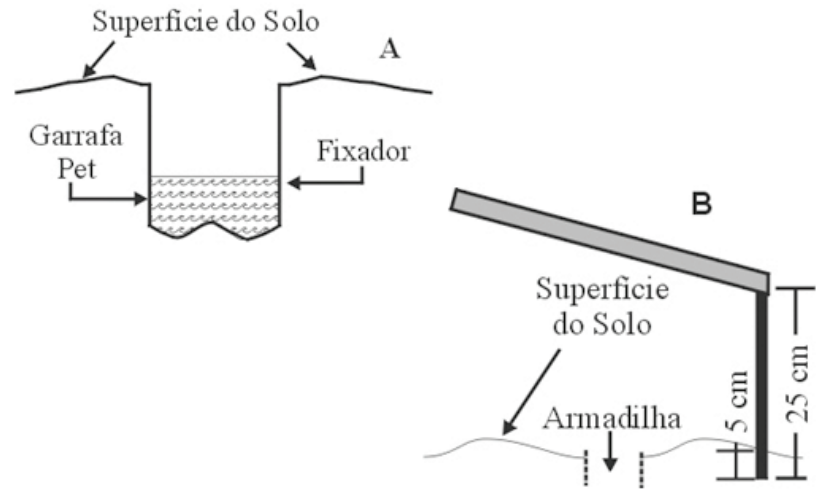

Figura 5. Armadilha de solo (tipo pitfall), adaptada de Majer (1978) e Castro et al. (1989). A - Armadilha de solo com garrafa Pet; B - Cobertura contra chuva a ser fixada acima da armadilha. 
Tabela 1. Características dos pontos de coleta: cobertura do dossel e altura de serrapilheira, em fragmento de mata atlântica pertencente ao Instituto Zoobotânico de Morro Azul, entre abril de 2008 e março de 2009.

\begin{tabular}{lcccc}
\hline Ponto & Composição Vegetal & Nível da & Altitude (m) & Luminosidade \\
$(\%)^{*}$
\end{tabular}

I Borda da mata, com vegetação intermediária.

I (o a $5 \mathrm{~cm})$

11,55

II Área de transição, com vegetação rasteira; aspecto descampado, predominância de gramínea Hypolitrum sp.

I (o a $5 \mathrm{~cm})$

655

46,84

Área intermediária, com vegetação rasteira; presença

III de arbusto e árvores (inclusive grande concentração de bambus).

IV Interior da mata (início da vegetação densa).

II (6 a $15 \mathrm{~cm})$

690

11,02

III $(>15 \mathrm{~cm})$

720

11,94

V Interior da mata (área central); vegetação de sub-bosque dominante com árvores espaçadas.

I (o a $5 \mathrm{~cm})$

6,82

VI

Interior da mata (área central)

III $(>15 \mathrm{~cm})$

676

4,38

VII Área intermediária, com pouca vegetação rasteira; presença de árvores grandes.

II $(6$ a $15 \mathrm{~cm})$

654

8,51

VIII

Área de transição, próxima à borda da mata; presença de árvores e arbustos.

II $(6$ a $15 \mathrm{~cm})$

640

6,46

* Esta porcentagem refere-se a taxa de não cobertura vegetal, ou seja, a diferença entre a área coberta e área não coberta em cada ponto.

\section{RESULTADOS E DISCUSSÃo}

Foram capturados 508 ortópteros durante as quatro coletas, sendo encontradas duas famílias, Gryllidae e Acrididae. A primeira, objeto deste estudo, representou 99,21\% dos ortópteros.

Abundância. Os grilídeos encontrados pertencem aos gêneros Anurogryllus (4,37\%), Argizala (3,98\%), Hygronemobius (19,88\%), Megalogryllus (15,11\%), Nemobius (29,62\%), Odontogryllus (6,76\%) e Pteronemobius (6,36\%). Destes insetos 70 indivíduos (13,92\%) não foram determinados por estarem nos primeiros instares de desenvolvimento (Tabela 2).

O gênero mais abundante foi Nemobius com 149 indivíduos, o que equivale a $29,62 \%$ do total de indivíduos. O gênero Argizala destaca-se como o menos abundante, ou seja, 20 espécimes, equivalendo a $3,98 \%$ da composição da fauna de Gryllidae. Quanto a ocorrência por pontos de coleta, verificou que Anurogryllus obteve maior e menor ocorrência no ponto IV $(31,82 \%)$ e ponto II (o\%), Argizala no ponto V (20\%) e no ponto II (5\%), Hygronemobius no ponto III (18\%) e ponto VIII ( $4 \%$ ), Megalogryllus no ponto I (25\%) e ponto V (3,95\%), Nemobius no ponto I $(24,16 \%)$ e ponto II $(3,36 \%)$, Odontogryllus no ponto I
$(35,29 \%)$ e ponto II (o\%) e Pteronemobius no ponto III $(28,13 \%)$ e no ponto II (o\%) (Tabela 2).

As abundâncias podem ser explicadas pela mobilidade de alguns gêneros como, por exemplo, Anurogryllus que apesar de terem preferência por locais descampados e relativamente degradados, não foi encontrado no ponto II, em nenhuma coleta, entretanto a mobilidade deste insetos SAmways \& Lockwood (1998), podem permitir um deslocamento para locais com mais recurso dentro do fragmento.

Dentre as áreas avaliadas, o ponto II foi o que apresentou menor abundância relativa de grilos $(4,77 \%)$, onde foi verificada a presença Megalogryllus, Hygronemobius, Nemobius e Argizala. Esta baixa abundância pode ser atribuída ao fato dessa ser uma área mais impactada, com menor cobertura vegetal no dossel, o que favorece o aumento da incidência solar e da umidade nesse micro habitat (McCluney \& Date 2008). Assim as condições e recursos necessários para ocorrência de alguns gêneros, limitam a sua presença neste ponto. Segundo Townsend et al. (2006) os competidores podem se excluir mutuamente ou poderão coexistirem, caso ocorra diferenciações ecológicas significativas de seus nichos realizados.

Tabela 2. Abundância de gêneros de grilídeos em fragmento de mata atlântica pertencente ao Instituto Zoobotânico de Morro Azul, entre abril de 2008 e março de 2009.

\begin{tabular}{|c|c|c|c|c|c|c|c|c|c|c|c|c|c|c|c|c|}
\hline \multirow{2}{*}{ Ponto } & \multicolumn{2}{|c|}{ Anurogryllus } & \multicolumn{2}{|c|}{ Argizala } & \multicolumn{2}{|c|}{ Hygronemobius } & \multicolumn{2}{|c|}{ Megalogryllus } & \multicolumn{2}{|c|}{ Nemobius } & \multicolumn{2}{|c|}{ Odontogryllus } & \multicolumn{2}{|c|}{ Pteronemobius } & \multicolumn{2}{|c|}{ NI } \\
\hline & $\mathrm{n}$ & $\%$ & $\mathrm{n}$ & $\%$ & $\mathrm{n}$ & $\%$ & $\mathrm{n}$ & $\%$ & $\mathrm{n}$ & $\%$ & $\mathrm{n}$ & $\%$ & $\mathrm{n}$ & $\%$ & $\mathrm{n}$ & $\%$ \\
\hline I & 2 & 9,09 & 2 & 10,0 & 15 & 15,0 & 19 & 25,0 & 36 & 24,16 & 12 & 35,29 & 1 & 3,13 & 9 & 12,86 \\
\hline II & o & 0,00 & 1 & 5,00 & 8 & 8,0 & 3 & 3,95 & 5 & 3,36 & 0 & 0,0 & o & 0,0 & 7 & 10,0 \\
\hline III & 2 & 9,09 & 3 & 15,0 & 18 & 18,0 & 14 & 18,42 & 32 & 21,48 & 2 & 5,88 & 9 & 28,13 & 16 & 22,86 \\
\hline IV & 7 & 31,82 & 2 & 10,0 & 22 & 22,0 & 7 & 9,21 & 21 & 14,09 & 8 & 23,53 & 6 & 18,75 & 12 & 17,14 \\
\hline $\mathrm{V}$ & 4 & 18,18 & 4 & 20,0 & 14 & 14,0 & 3 & 3,95 & 12 & 8,05 & 3 & 8,82 & 1 & 3,13 & 8 & 11,43 \\
\hline VI & 3 & 13,64 & 2 & 10,0 & 14 & 14,0 & 7 & 9,21 & 15 & 10,07 & 3 & 8,82 & 5 & 15,63 & 6 & 8,57 \\
\hline VII & 1 & 4,55 & 3 & 15,0 & 5 & 5,0 & 10 & 13,16 & 18 & 12,08 & 2 & 5,88 & 5 & 15,63 & 7 & 10,0 \\
\hline VII & 3 & 13,64 & 3 & 15,0 & 4 & 4,0 & 13 & 17,11 & 10 & 6,71 & 4 & 11,76 & 5 & 15,63 & 5 & 7,14 \\
\hline Total & 22 & 15,11 & 20 & 4,37 & 100 & 19,88 & 76 & 15,11 & 149 & 29,62 & 34 & 6,76 & 32 & 6,36 & 70 & 13,92 \\
\hline
\end{tabular}

NI= Não identificados 
Esse resultado, menor abundância, sugere que estes gêneros podem ocorrer em locais com maior luminosidade e pouca umidade e menor nível de serrapilheira. Devendo levar em conta também a pouca disponibilidade de abrigo (tronco das árvores e serapilheira) e alimentos para esses insetos, uma vez que a estrutura do habitat e a disponibilidade de recursos podem limitar a diversidade local (Ricklefs \& Shuluter 1993). Desta forma e apesar da ocorrência nos demais pontos de coleta, os gêneros acima citados, possui certa adaptabilidade a condições adversas e em locais de baixa disponibilidade de recursos.

Os pontos I e III foram os que apresentaram maior abundância relativa de grilos (19,09\% cada ponto), seguido do ponto IV (16,90\%). Apesar de serem três áreas distintas em relação à composição vegetal e nível de serrapilheira, estas se mostraram semelhantes em relação à fauna de grilos. Mesmo que a fauna de grilos seja afetada pela umidade (McClunEY \& DATE 2008) e perturbação do habitat (SPERBER et al. 2007).

Segundo WARREN \& Zou (2002), a diversidade vegetal oferece diferentes recursos alimentares o que influencia na quantidade da serapilheira ingerida pela fauna do solo controlando assim o índice de abundância dos organismos em um local.

A serrapilheira pode fornecer recurso alimentar para grilos, como folhas recém-caídas, flores, fungos e frutos (GANGWERE 1961) e também representar abrigo e espaço livre dos inimigos (SzINWELSKI et al. 2009). Porém, neste estudo, o nível de serapilheira parece não interferiu na abundância absoluta de grilos, pois os dois pontos (IV e VI) com maior nível de serapilheira não foram os que mais apresentaram amostras desses insetos. Ainda em relação à quantidade de serapilheira, os três pontos que apresentaram maior abundância absoluta de grilos estão em três níveis distintos na quantidade de serapilheira (Figura 6).

O pico populacional foi observado na coleta IV (verão), período mais quente do ano, com 165 indivíduos coletados, em contrapartida, no inverno (coleta II) houve menor abundância absoluta, com 102 grilos coletados (Figura 7). Sabendo que a sazonalidade de insetos depende em grande parte de variações climáticas (Wolda 1978), esses ortópteros possuem afinidade com climas mais quentes e úmidos. E o aumento do índice pluviométrico dessa estação quente parece favorecer a abundância desses insetos, uma vez que ciclos sazonais de insetos tropicais têm sido atribuídos à variação na disponibilidade de recursos, provocada pela alternância entre períodos secos e chuvosos (NEves et al. 2010).

Os grilos possuem padrões de ciclo de vida controlados pelo fotoperiodismo e variações térmicas, que no caso das regiões tropicais, possuem dinâmica de reprodução e crescimento contínuo, tendo seus estágios de desenvolvimento presente em todas as estações do ano, diferente do que é observado em climas temperados (ZeFa et al., 2006). Entretanto MASAKi \& Walker (1987) em seus estudos afirma que o controle do fotoperíodo combinado com a mudança sazonal da temperatura garantiria hibernação ninfas, em climas temperados.

Similaridade. Os pontos V, VI, VII e VIII, foram os mais similares, segundo a análise de cluster (Figura 8). Apesar das diferentes altitudes, estes possuem estrutura vegetal similar em estrutura, com maior cobertura de dossel, pouca vegetação de sub-bosque e nível serrapilheira, maior que os demais pontos. Isso mostra um equilíbrio na composição da fauna de grilos, pois a variação do número de indivíduos foi menor nesses pontos. Esse equilíbrio pode estar relacionado à competição por nicho, predação, abrigo, etc., com outros insetos. Esses resultados também podem indicar que esses insetos são mais resistentes às pequenas variações de habitat quando comparados com algumas famílias de outras ordens (FERNANDEs et al. 2011a) em estudos com coleópteros no mesmo local.

O dendograma de similiridade mostrou haver menor relação dos insetos capturados nas coletas I e II, respectivamente, quase não havendo diferença na abundância de grilos (Figura 9). Enquanto que nas coletas III e IV o número de insetos foi maior, visto que a sazonalidade é um fator que interfere diretamente relacionado à abundância de insetos (WOLDA 1978).

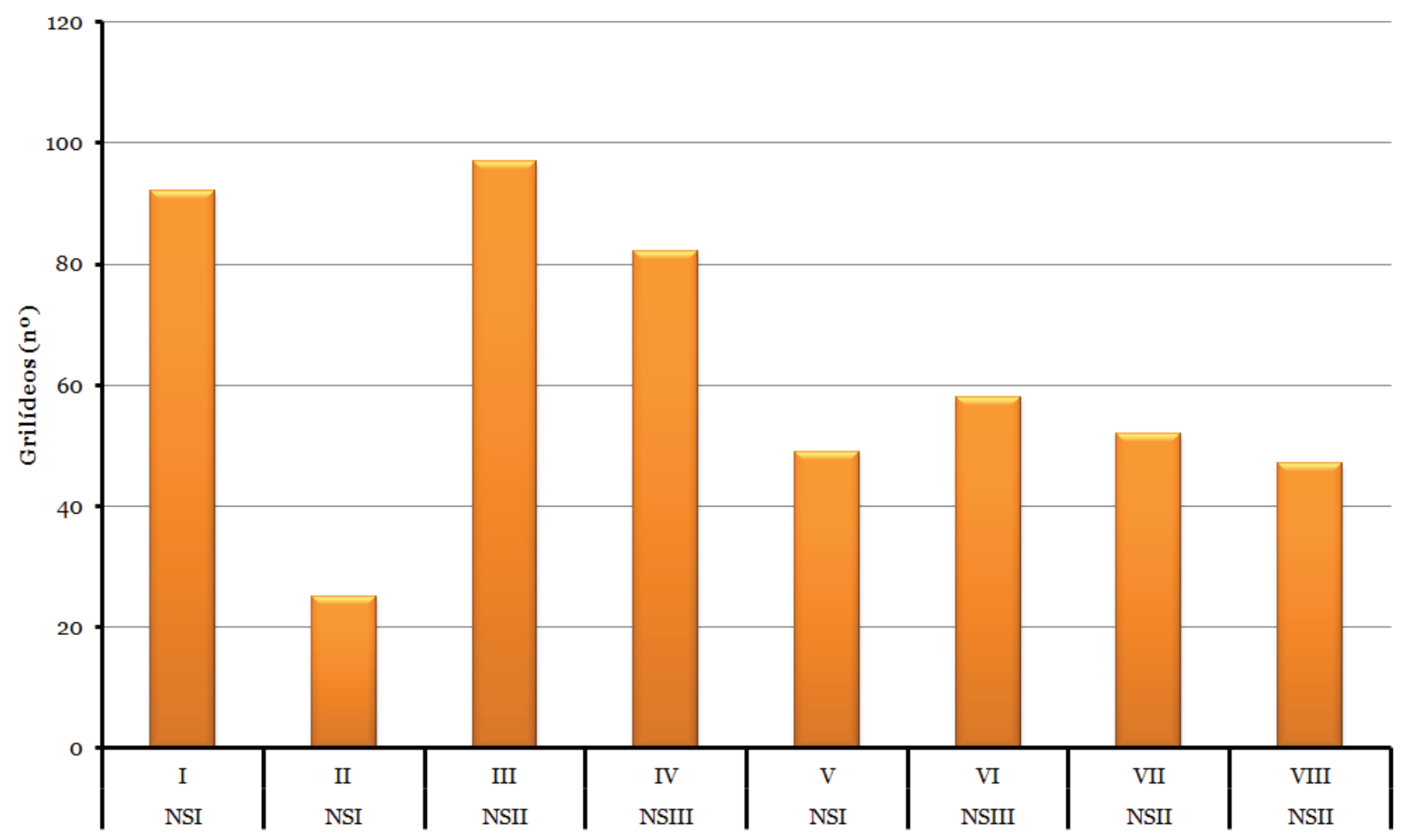

Ponto de Coleta/ Nível de Serapilheira

Figura 6. Abundância absoluta de grilos em relação ao nível de serapilheira somada todas as coletas, no período de abril de 2008 e março de 2009 , em fragmento de floresta pluvial atlântica pertencente ao Instituto Zoobotânico de Morro Azul. 


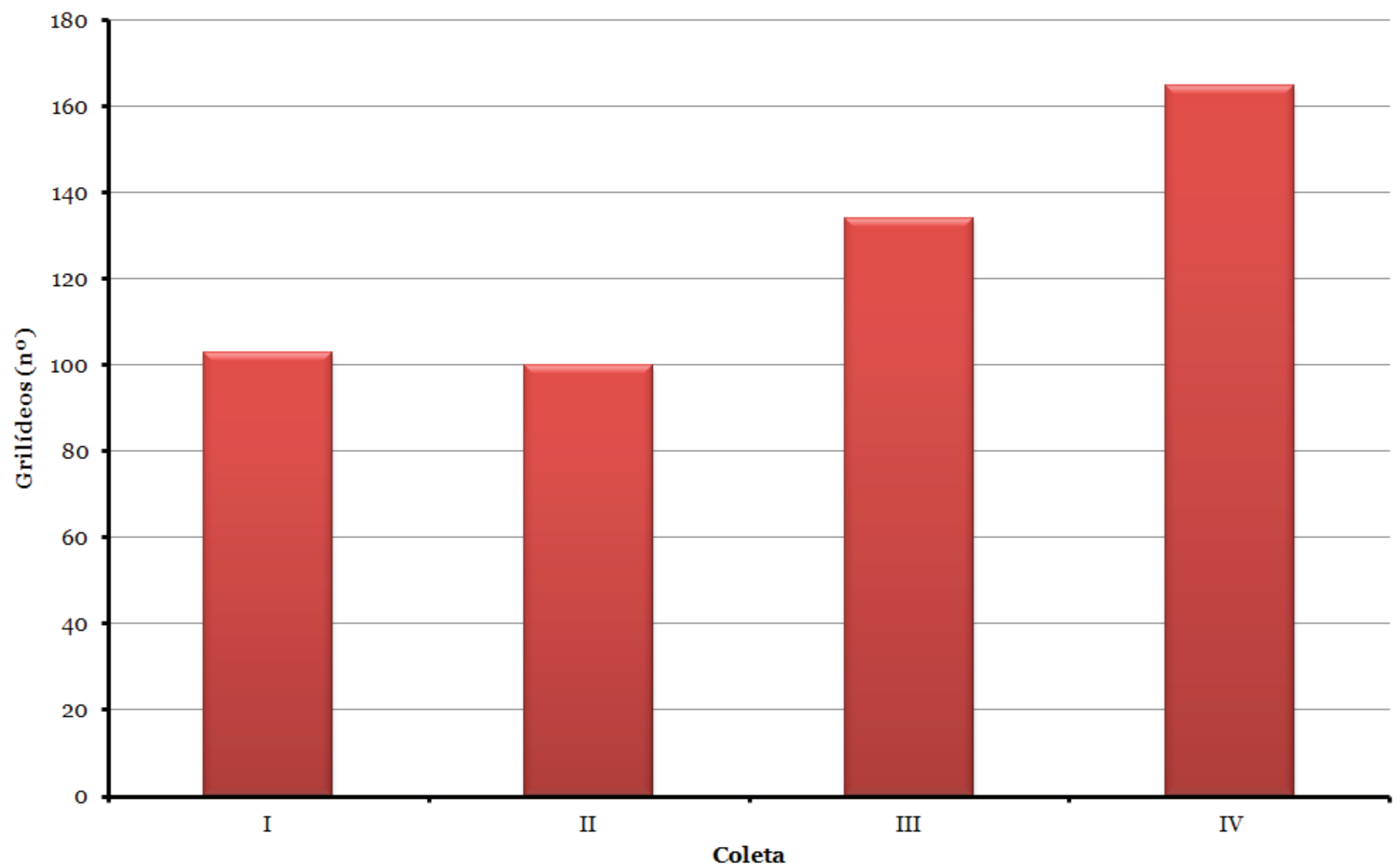

Figura 7. Abundância absoluta de grilos em relação às quatro estações do ano, no período de abril de 2008 e março de 2009, em fragmento de floresta pluvial atlântica pertencente ao Instituto Zoobotânico de Morro Azul.

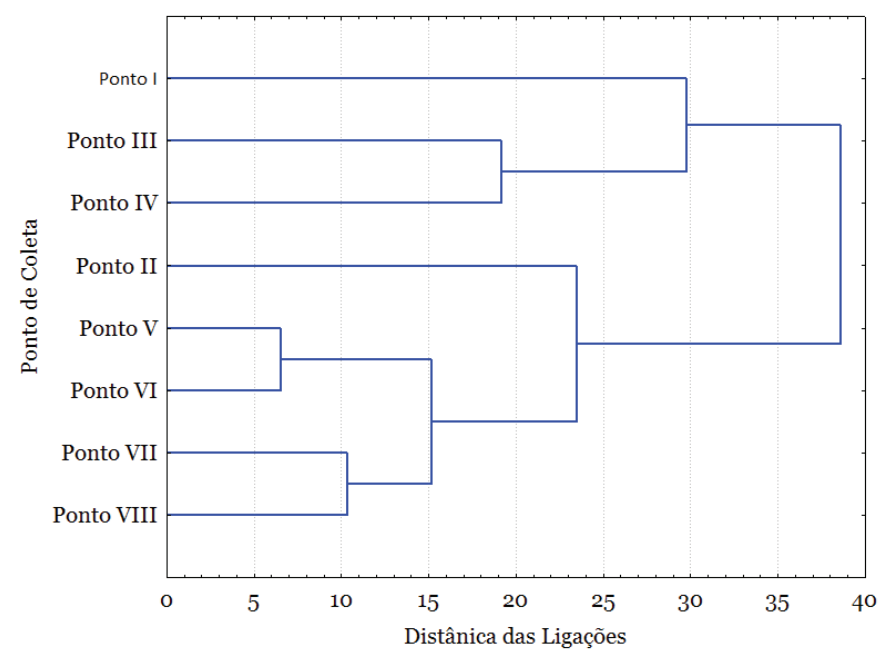

Figura 8. Diagrama de árvore da família Gryllidae entre os oito pontos de coleta, baseado na análise Cluster (Complete Linkage), no período de abril de 2008 e março de 2009, em fragmento de floresta pluvial atlântica pertencente ao Instituto Zoobotânico de Morro Azul.

Diversidade, Dominância e Equitabilidade de Gêneros.

A primeira coleta (107 indivíduos coletados) apresentou índice de Diversidade de Shannon H' = o,860, enquanto a mais abundante, a coleta de verão (IV), apresentou o menor índice, com H' = o,6534 ( $n=165)$, sendo que ocorre dominância de Nemobius $(n=38)$ (Figura 10). Segundo o índice de Simpson, apresentando Ds $=0,7370$, diferente do que ocorre na coleta I $(\mathrm{Ds}=0,8600)$. A coleta II (102 indivíduos) apresentou H' = 0,7660 para Shanon e Ds $=0,7855$ para Simpson. A coleta II verificou-se H' = 0,7139 e Ds $=0,7723$ (Figura 10).

Conforme seiniciam as estações mais chuvosas, apesar do aumento na abundância, ocorreram menores valores de diversidade. Embora ocorra a redução na abundância nas estações mais secas (WoldA 1977), alguns grupos podem reduzir sua abundância

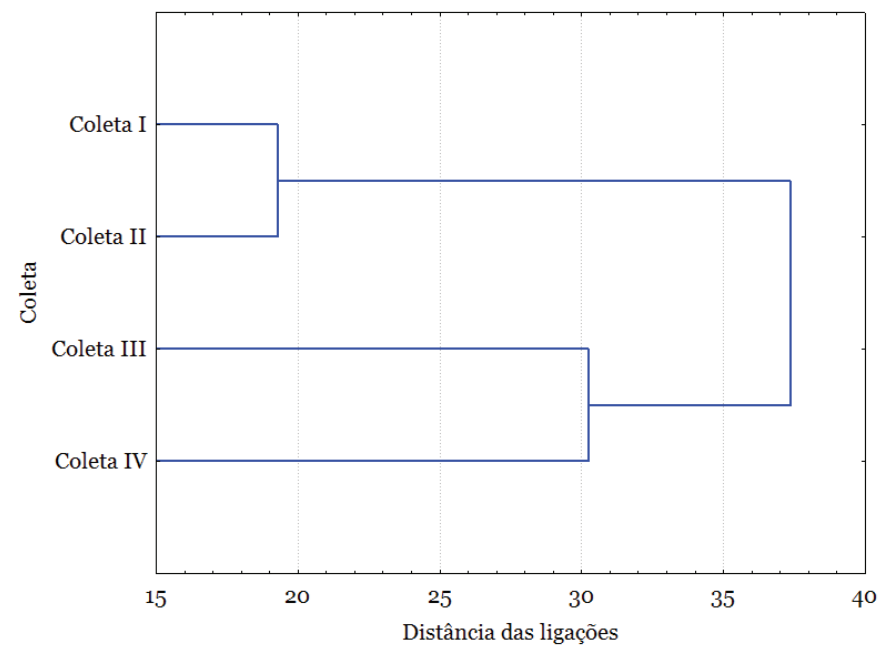

Figura 9. Diagrama de árvore da família Gryllidae nas quatro coletas, abrangendo as quatro estações do ano, baseado na análise Cluster (Complete Linkage), no período de abril de 2008 e março de 2009, em fragmento de floresta pluvial atlântica pertencente ao Instituto Zoobotânico de Morro Azul.

nas estações chuvosas (FERNANDEs et al. 2011b). Sendo assim, a ausência de Anurogryllus na primavera e verão e Argizala apenas no verão sugerem que estes gêneros têm preferência por clima mais seco. A ausência destes gêneros interferiu nos valores dos índices de diversidade, tanto em Shanon quanto em Simpson.

Ao analisar os índices de diversidade por ponto, observamos que os valores aumentam conforme a complexidade do ambiente e nível de serrapilheira aumenta. Assim como no que diz respeito à sazonalidade, a abundância não foi um fator determinante para a diversidade nos pontos, tanto em Shannon quanto em Simpson.

No ponto VIII obteve-se os maiores valores de Shannon ( $\mathrm{H}^{\prime}$ $=0,8391$ ) e Simpson (Ds = o,8511), local onde ocorre menor abundância. Como ocorreu menor dominância de gêneros, este 
resultado pode sugerir que os grilos encontrados neste ponto tem preferência por áreas com maior incidência de luz, porém, com nível de serrapilheira maior (Figuras 6,11 ).

Entretanto, no ponto II, o nível de serrapilheira pode ter interferido na diversidade $\left(\mathrm{H}^{\prime}=0,6274\right.$ e $\left.\mathrm{Ds}=0,7754\right)$ assim como na abundância, corroborando que os grilos não possuem afinidade com locais descampados. A ocorrência de apenas quatro gêneros interferiu no valor da diversidade no local. A ausência dos gêneros Pteronemobius, Odontogryllus e Anurogryllus pode indicar que estes gêneros possuem mais sensibilidade a locais degradados, apesar da literatura afirmar que Anurogryllus é uma espécie de locais mais abertos (SAMWAYS \& LOCKWOOD 1998).

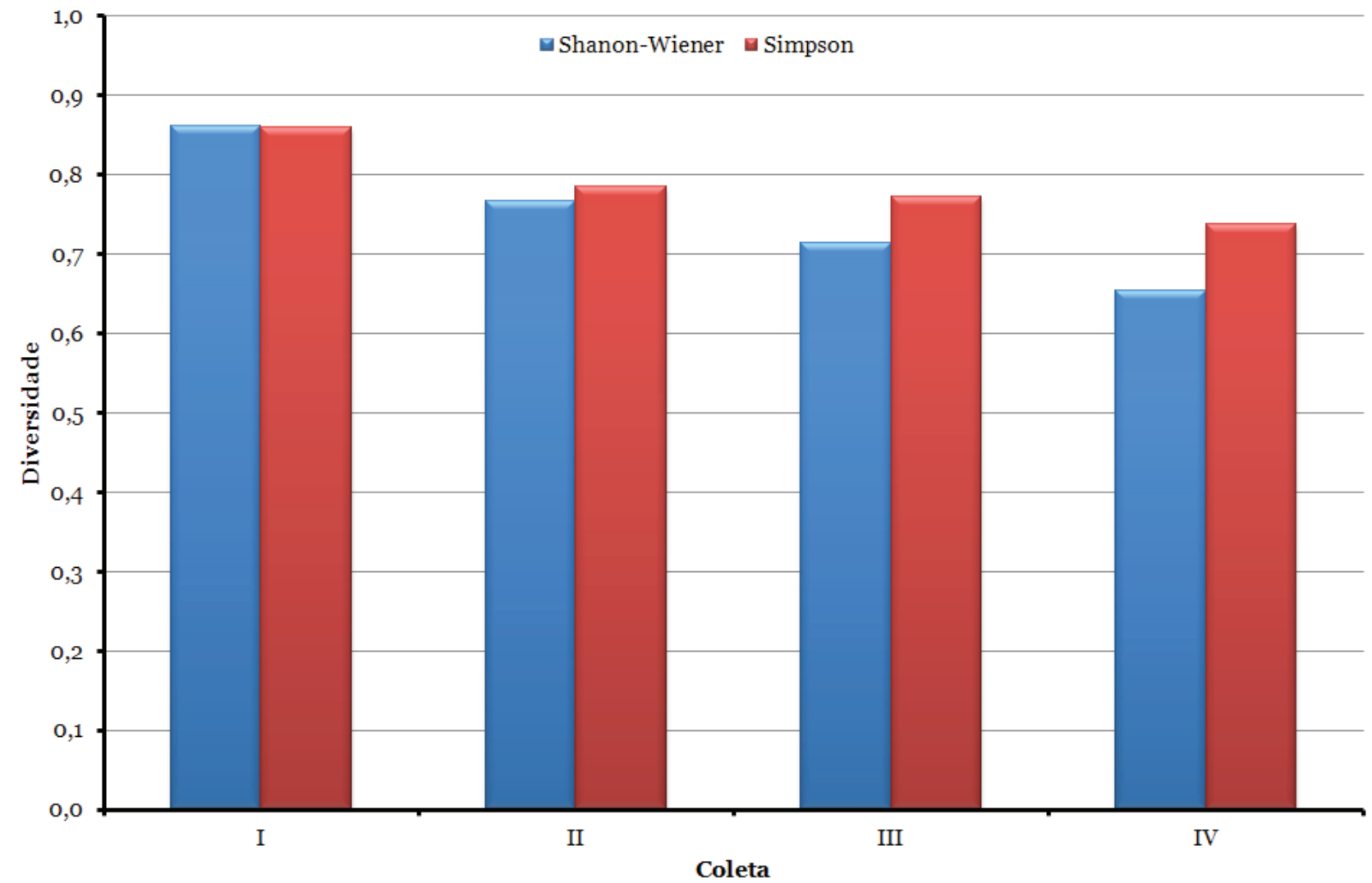

Figura 10. Diversidade de Shannon (H’) e Simpson (Ds), nas quatro coletas anuais, no período de abril de 2008 e março de 2009, em fragmento de floresta pluvial atlântica pertencente ao Instituto Zoobotânico de Morro Azul.

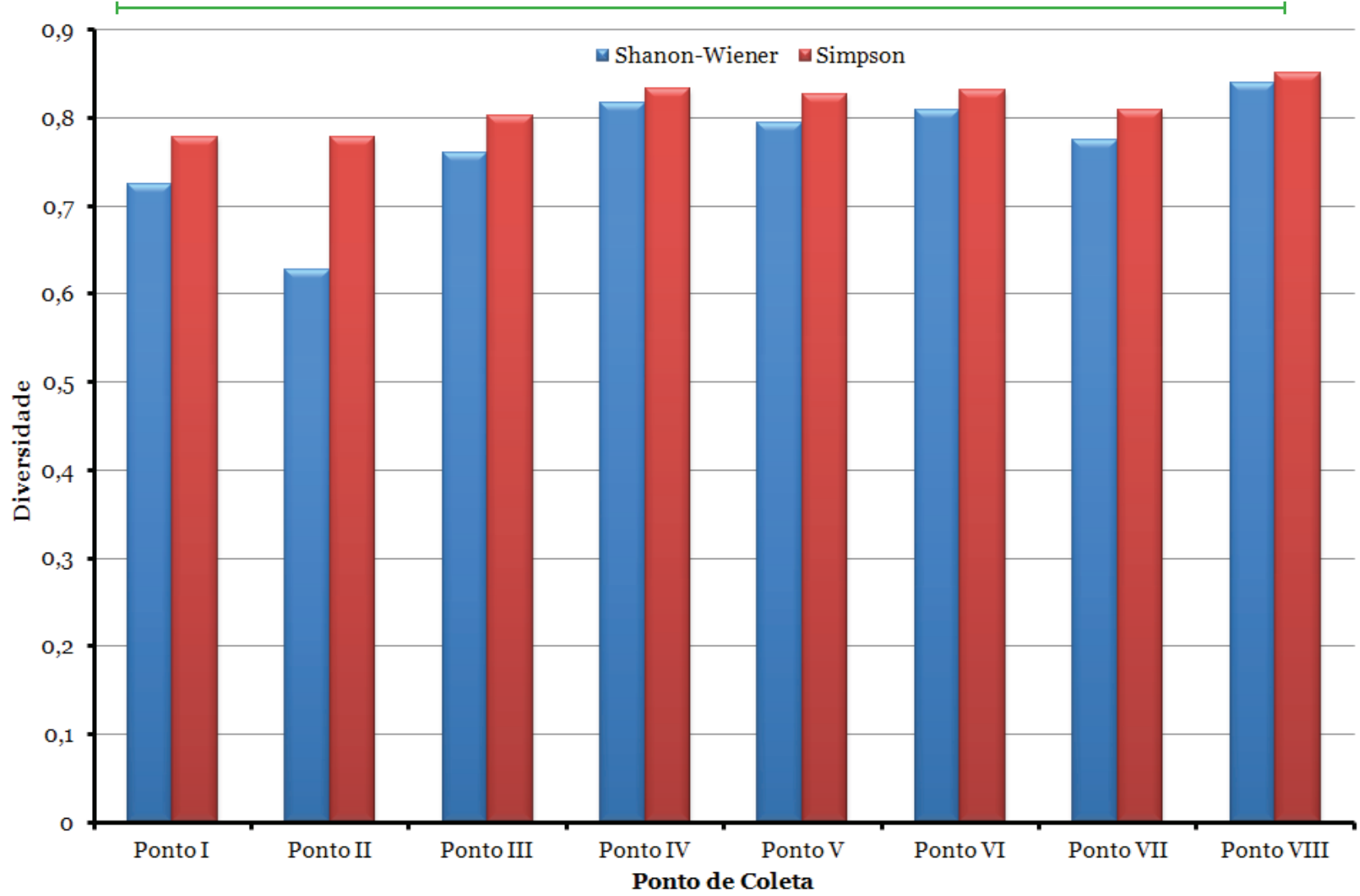

Figura 11. Diversidade de Shannon (H') e Simpson (Ds) em relação aos pontos de coleta, em diferentes complexidades de ambiente, nas quatro coletas anuais, no período de abril de 2008 e março de 2009 em fragmento de floresta pluvial atlântica pertencente ao Instituto Zoobotânico de Morro Azul. 
Em seus estudos Alexander \& Thomas (1959) afirmaram que algumas espécies do gênero Nemobius apresentam preferência por locais mais secos e descampados, o que explica a maior dominância deste gênero nos pontos I $\left(\mathrm{H}^{\prime}=0,7249\right.$ e $\left.\mathrm{Ds}=0,7785\right)$ e III $\left(H^{\prime}=0,7604\right.$ e Ds=0,8024) (Figura 11).

É de se esperar que a Equitabilidade $J$ e H' se comportam inversamente à dominância, uma vez que os valores altos destes dois índices indicam uma baixa concentração de dominância (ODum 2001), mas como visto, este fato ocorrerá caso exista apenas uma espécie dominante, pois os modelos matemáticos atuais não conseguem prever a co-dominância de espécie (RODRIGUEs et al. 2008).

Como parte do componente de diversidade a equitabilidade e diretamente proporcional à diversidade e da mesma forma inversamente proporcional à dominância (RoDRIGUEs et al. 2009). Desta forma, em populações onde há competição por nicho está regra poderá ser considerada como verdadeira, pois maior diversidade significa cadeias alimentares maiores e maior caos de simbiose (mutualismo, predatismo, etc.) (ODUm 2001).

Os dados evidenciam que as modificações na cobertura vegetal promovem alterações na abundância e diversidade de grilos, pois eles mostraram maior sensibilidade em áreas descampadas, diminuindo consideravelmente sua população no ponto mais impactado. Podem-se chamar os grilos de indicadores ambientais, uma vez que estes insetos responderam às perturbações do meio.

O nível de serapilheira não interferiu na abundância de grilos. Esperava-se que eles obtivessem maior amostragem no maior nível, mas não foi o encontrado. Em relação à sazonalidade, esses insetos responderam positivamente na estação do verão, com período mais chuvoso e clima mais quente, entretanto, foi inversamente proporcional à diversidade, que caiu conforme as estações quentes se aproximavam, sugerindo que certos gêneros podem ter preferência por estações mais secas e frias.

A diversidade respondeu positivamente em relação à cobertura do dossel, apresentando maiores valores conforme a complexidade do ambiente aumenta, embora com a abundância não tenhamos a mesma resposta.

São escassos estudos no Brasil sobre a ordem Orthoptera, principalmente em áreas que não sejam agrícolas. Este estudo buscou agregar informações sobre os grilos, pois é um grupo que apresenta poucas pesquisas e contribuiu para o melhor conhecimento da biodiversidade presente na região, pois pouco se conhece a fauna desses insetos neste fragmento de Mata Atlântica no interior do estado do Rio de Janeiro.

\section{AGRADECIMENTO}

Ao Instituto Zoobotânico de Morro Azul, na pessoas do seu diretor-presidente o prof. M.Sc. Hélio Freitas Santos, por conceder autorização para realização do presente estudo.

\section{REFERÊNCIAS}

Alexander, R. \& E.S. Thomas, 1959. Systematic and behavioral studies on the crickets of the Nemobius Fasciatus group (Orthotera: Gryllindae: Nemobiinae). Entomological Society of America. Annals of the Entomological Society of America, 52: 591-605.

Basset, Y., V. Novotny, S.E. Miller \& R.L. Kitching, 2003. Arthropods of tropical forest spatio-temporal dynamics and resource in the canopy. Cambridge: Cambridge University Press. 490p.

Castro, A.G., M.V.B. Queiroz \& L.M. Araújo, 1989. Estrutura e diversidade de comunidades de formigas em pomar de citros. Anais da Sociedade Entomológica do Brasil, 18: 229-246.

Chopard, L., 1938. La biologie des Orthoptères. Encyclopedie
Entomologique, Ser. A 20: 1-541.

Chopard, L., 1956. Some crickets from South America (Gryllidea and Tridactyloidea). Proceedings of the United States National Museum, 106: 241-293.

Eades, D.C., D. Otte, M.M. Cigliano \& H. Braun, 2013. Orthoptera Species File Online Version 5.0/5.o. Acessível em: <http:// orthoptera.speciesfile.org>.

Fernandes, F.S., S.S. Alves, H.F. Santos \& W.C. Rodrigues, 2011a. Staphylinidae e Silphidae (Coleoptera) como Potenciais Famílias Bioindicadoras de Qualidade Ambiental. Revista Eletrônica Teccen, 4: 17-32.

Fernandes, M.L., M.R. Pereira \& C.F. Sperber, 2011b. Efeitos da sazonalidadenaabundância degrilos(Orthoptera:Grylloidea). X Congresso Brasileiro de Ecologia, São Lourenço/MG, 1-4p.

Frazer, G.W., C.D. Canham, \& K.P. Lertzman, 1999. Gap Light Analyzer (GLA), Version 2.0: Imaging software to extract canopy structure and gap light transmission indices from true-colour fisheye photographs, users manual and program documentation. Acessível em: <http://www.rem.sfu.ca/ forestry/publications/downloads/gaplightanalyzer.htm >.

Freitas, A.V., R.B. Francini \& K.S. Brown JunioR, 2004. Insetos como indicadores ambientais. p. 125-151. In: Cullen Junior, L., R. Rudran \& C. Valladares Pádua (Orgs.). Biologia da Conservação \& Manejo da Vida Silvestre. Curitiba: Editora UFPR. 652p.

Gangwere, S.K., 1961. A monograph on food selection in Orthoptera. Transactions of the American Entomological Society, 87: 67-230.

Greenberg, C.H. \& T.G. Forrest, 2003. Seasonal abundance of ground - dwelling arthropods in forest and canopy gaps of the southern Appalachians. Southeastern Naturalist, 2: 591-608.

Huber, F., T.E. Moore \& W. Lower, 1989. Cricket behavior and neurobiology. Cornell University Press, Ithaca and London, $565 p$.

Lima-Ribeiro, M.S., 2008. Efeitos de borda sobre a vegetação e estruturação populacional em fragmentos de Cerradão no Sudoeste Goiano, Brasil. Acta Botânica Brasilica, 22: 535545 .

Lutinski, J.A. \& F.R.M. Garcia, 2005. Análise faunística de Formicidae (Hymenoptera: Apocrita) em ecossistema degradado no município de Chapecó, Santa Catarina. Biotemas, 18: 73-86.

Majer, J.D., 1978. An improved pitfall trap for sampling ants and other epigaeic invertebrates. Journal of Australian Entomology Society, 17: 261-262.

Masaki, S. \& T.J. Walker. 1987. Cricket life cycles. Evolutionary Biology, 21:349-423

Matyot, P., 1998. The orthopteroids of the Seychelles: A threatened island fauna. Journal of Insect Conservation, 2: 235-246.

McCluney, K.E. \& R.C. Date, 2008. The effects of hydration on growth of the house cricket, Acheta domesticus. Journal of Insecta Science, 8: 1-9.

Mews, C.M., 2006. Os Grilos Phalangopsidae (Orthoptera: Grylloidea) em Viçosa, Minas Gerais, Brasil. Dissertação (Mestrado em Entomologia) - Universidade Federal de Viçosa. 105p. Disponível em: <http://www.tede.ufv. br/tedesimplificado/tde arquivos/11/TDE-2007-0313To63858Z-403/Publico/texto\%20completo.pdf >.

Murphy, P.G. \& E. Lugo, 1986. Ecology of tropical dry forest. Annual Review of Ecology and Systematics, 17: 67-88.

Neves, F.S., L.S. Araújo, M.M. Espírito-Santo, M. Fagundes \& G.W. Fernandes, 2010. Efeito da estratificação florestal e da sucessão secundária sobre a fauna de insetos herbívoros associada ao dossel de uma floresta estacional decidual, Biota, 3: 33-44.

Odum, E.P., 2001. Fundamentos de Ecologia. $6^{\mathrm{a}}$ ed. Lisboa: Fundação Calouste Gulbenkian, 927p.

Pereira, M.R., 2012. Sistemática de Nemobiinae (Orthoptera, Grylloidea, Trigonidiidae). Tese (Doutorado em Entomologia) - Universidade Federal de Viçosa, 95p.

Pezzini, F.F., D.O. Brandão, B.D. Ranieri, M.M. Espírito-Santo, 
C.M. Jacobi \& G.W. Fernandes, 2008. Polinização, dispersão de sementes e fenologia de espécies arbóreas no Parque Estadual da Mata Seca. MG. Biota, 1: 37-45.

Ricklefs, R.E. \& D. Schluter, 1993. Species diversity: regional and historical influences, p. 350-363. In: Ricklefs RE \& D. Schluter (eds) Species diversity in ecological communities: historical and geographical perspectives. University of Chicago Press, Chicago, 416p.

Rodrigues, W.C., 2005. DivEs - Diversidade de espécies. Versão 2.o. Software e Guia do Usuário. Disponível em: < $\underline{\text { http:// }}$ www.ebras.bio.br/dives>.

Rodrigues, W.C., P.C.R. Cassino \& R. Silva Filho, 2008. Ocorrência e Distribuição de Coccinelídeos (Coleoptera, Coccinellidae) Associados às Plantas Cítricas no Estado do Rio de Janeiro. EntomoBrasilis, 1: 23-27.

Rodrigues, W.C., P.C.R. Cassino, M.V. Spolidoro \& R. SilvaFilho, 2009. Insetos Sugadores (Sternorrhyncha) em Cultivo Orgânico de Tangerina cv. Poncã (Citrus reticulata Blanco): Diversidade, Constância, Freqüência e Flutuação Populacional. EntomoBrasilis, 2: 42-48.

Samways, M.J. \& J.A. Lockwood, 1998. Orthoptera conservation: pests and paradoxes. Journal of Insect Conservation, 2: 143149.

Sánchez-Azofeita, G.A., M. Quesada, J.P. Rodríguez, J.M. Nassar, K.E. Stoner, A. Castillo, T. Garvin, E.L. Zent, J.C. CalvoAlvarado, M. Kalascka, L. Fajardo, J.A. Gamon \& P. CuevasReyes, 2005. Research Priorities for Neotropical Dry Forests. Biotropica, 37: 477-485.

Silveira Neto, S., R.C. Monteiro, R.A. Zucchi \& R.C.B. Moraes, 1995. Uso da análise faunística de insetos na avaliação do impacto ambiental. Scientia Agrícola, 52: 9-15.

Sperber, C.F, L.G.S. Soares \& M.R Pereira, 2007. Litter disturbance and trap spatial positioning affects number of captured individuals and genera of crickets (Orthoptera: Grylloidea). Journal of Orthoptera Research, 16:1-7.

Sperber, C.F., 1999. Por que há mais espécies de grilos (Orthoptera: Grylloidea) em fragmentos florestais maiores? Dissertação - Universidade Estadual Paulista Julio de Mesquita Filho, UNESP, Rio Claro. 295p.

Sperber, C.F., G.H. Vieira \& M.H. Mendes. 2003. Aprimoramento da amostragem de grilos de serapilheira (Orthoptera:
Gryllidae) por armadilha. Neotropical Entomology, 32: 733 -735 .

Strohecker, H.F., 1953. The Gryllacrididae and Gryllidae of The Bahama Islands, British West Indies (Orthoptera). American Museum Novitates, $n^{0} 1618,11 p$.

Szinwelski, N., 2009. Riquezas de Espécies de Grilos (Orthoptera: Grylloidea) em fragmentos de Mata Atlântica em regeneração. Dissertação (Mestrado em Biologia Animal) - Universidade Federal de Viçosa. 37p., Disponível em: <http://www.tede. ufv.br/tedesimplificado/tde arquivos/29/TDE-2009-1204Tog0123Z-2076/Publico/texto\%20completo.pdf>

Tabanez, A.J., V.M. Viana \& A.S. Dias, 1997. Conseqüências da fragmentação e do efeito de borda sobre a estrutura, diversidade e sustentabilidade de um fragmento de Floresta de Planalto de Piracicaba. Revista Brasileira de Biologia, 57: 47-60.

Thomanzini, M.J. \& A.P.B.W. Thomanzini, 2000. A fragmentação florestal e a diversidade de insetos nas florestas tropicais úmidas. Rio Branco: EMBRAPA Acre, (Circular Técnica, 57), 21p.

Townsend, C.R., M. Begon \& J.L. Harper, 2006. Fundamentos em Ecologia. ${ }^{\mathrm{a}}$ ed., Porto Alegre: Artmed, 592p.

Warren, M.W. \& X. Zou, 2002. Soil macrofauna and litter nutrients in three tropical tree plantations on a disturbed site in Puerto Rico. Forest Ecology and Management, 170: 161171.

Wolda, H., 1977. Fluctuations in abundance of some Homoptera in a neotropical forest. Geo-Eco-Trop, 3: 229 - 257.

Wolda, H., 1978. Seasonal fluctuations in rainfall, food, and abundance of tropical insects. Journal of Animal Ecology, 47: 369-381.

Zefa, E., F.M. Rúbio, A.R. Rinald, L.H. Gollin, D.B.F. Silva \& P.G.B.S Dias, 2006. Seasonal life cycle of the tropical cricket Eneoptera surinamensis. Iheringia, Série Zoológica, 96: 267269.

\section{Recebido em: 21/o7/2013}

Aceito em: 27/10/2013

\section{Como citar este artigo:}

Oliveira, C.S.P., M.P. Mendes, M.N. Duarte \& W.C. Rodrigues , 2013. Composição e Diversidade da Fauna de Grilos (Orthoptera: Grylloidea) em um Fragmento de Floresta Pluvial Atlântica do Estado do Rio de Janeiro. EntomoBrasilis, 6(3): 184-192.

Acessível em: http://www.periodico.ebras.bio.br/ojs/index.php/ebras/article/view/374. doi:10.12741/ebrasilis.v6i3.374
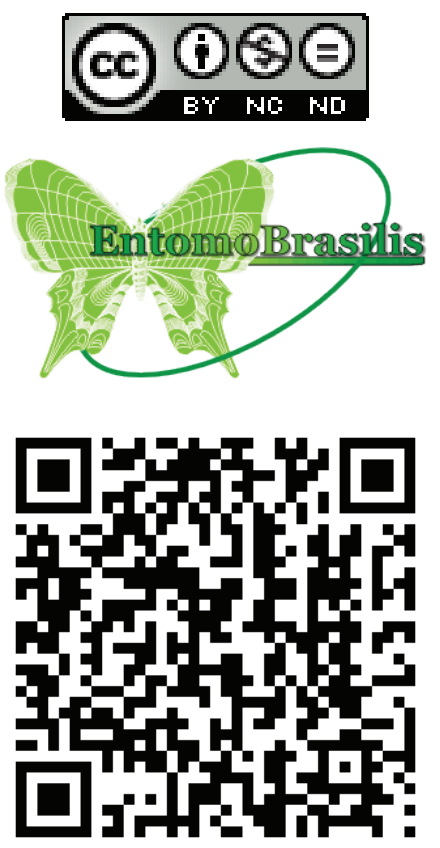